\title{
EFEITO DA TÉCNICA DE CONTRASTE SOBRE A CONCENTRAÇÃO PLASMÁTICA DE LACTATO DURANTE A RECUPERAÇÃO PÓS-ESFORÇO
}

\author{
Heliard Rodrigues Dos Santos Caetano ${ }^{1}$, Guilherme Oliveira Martins de Lima ${ }^{1}$, Guilherme Akio Tamura \\ Ozaki ${ }^{2}$, Francis Lopes Pacagnelli ${ }^{1}$, Robson Chacon Castoldi ${ }^{1}$, Everton Alex Carvalho Zanuto ${ }^{1}$
}

Universidade do Oeste Paulista - UNOESTE, Curso de Educação Física, Presidente Prudente, SP. ${ }^{2}$ Universidade Estadual de Campinas - UNICAMP, Campinas, SP.E-mail: everton@unoeste.br

\section{RESUMO}

O objetivo do estudo foi analisar a técnica de contraste sobre a concentração plasmática de lactato durante a recuperação pós-exercício de atletas de voleibol. Foram voluntárias deste estudo 18 jogadoras de voleibol, com idade entre 17 e 18 anos, distribuídas de forma aleatória em dois grupos, sendo estes: Grupo controle (GC) e Grupo Tratado (GT). O GC foi submetido a um protocolo de indução à hiperlactacidemia e, após este processo, permaneceram sentadas em durante $25 \mathrm{~min}$. O GT passou pelo mesmo protocolo, porém, foram submetidas ao tratamento de contraste por $16 \mathrm{~min}$. Observou-se nos grupos GC e GT os respectivos valores de lactato nos momentos repouso, PÓS-5, PÓS-15 e PÓS- 25 min de esforço: 1,97 $\pm 0,3$ vs $1,63 \pm 0,6 \mathrm{mmol} / \mathrm{L} ; 8,75 \pm 1,5$ vs $7,35 \pm 4,1 \mathrm{mmol} / \mathrm{L} ; 8,53 \pm 1,6$ vs $5,3 \pm 1,9 \mathrm{mmol} / \mathrm{L}$ e $6,87 \pm 2,1 \mathrm{mmol} / \mathrm{L}$ vs $3,33 \pm 1,47 \mathrm{mmol} / \mathrm{L}$. Conclui-se que a técnica de contraste se mostrou eficiente para a remoção do lactato em jogadoras de voleibol.

Palavras-chave: exercício, teste de esforço, ácido láctico, imersão, crioterapia.

\section{EFFECTS OF CONTRAST TECHNIQUE ON PLASMATIC LACTATE CONCENTRATION DURING POST-EFFORT RECOVERY}

\begin{abstract}
The aim of this study was to analyze the effects of the contrast technique on lactate plasmatic concentration during post-exercise recovery in volleyball athletes. For this study, 18 volleyball players between 17 and 18 years old were included. The volunteers were randomly divided into two groups, Control Group (CG) and Treated Group (TG). The CG was submitted to a hyperlactacidemic induction protocol, immediately after which they remained seated at rest for $25 \mathrm{~min}$. The TG performed the same protocol and then they were submitted to a contrast treatment for $16 \mathrm{~min}$. The following respective lactate values were observed in the CG and TG groups at the moments rest, POST-5, POST-15 and POST-25 min of effort: $1.97 \pm 0.3$ vs $1.63 \pm 0.6 \mathrm{mmol} / \mathrm{L} ; 8.75 \pm 1.5$ vs $7.35 \pm 4.1 \mathrm{mmol} / \mathrm{L} ; 8.53 \pm 1.6$ vs $5.3 \pm 1.9 \mathrm{mmol} / \mathrm{L}$ and $6.87 \pm 2.1$ vs $3.33 \pm 1.47 \mathrm{mmol} / \mathrm{L}$. It was concluded that the contrast technique was efficient to remove blood lactate in volleyball players.
\end{abstract}

Keywords: Exercise, Exercise Test, Lactic Acid, Immersion, Cryotherapy.

\section{INTRODUÇÃO}

O exercício físico é definido como uma variedade de atividades físicas, que diferem largamente quanto à sua intensidade, duração e capacidades exigidas para sua prática, caracterizando-se por intervenção que promove alteração na homeostase ${ }^{1,2}$. De acordo com Forjaz e Tricoli $^{3}$ e Barnett ${ }^{4}$, as mudanças que constituem adaptações não possuem apenas caráter agudo, mas também crônico, que tornam possível a realização do exercício físico, mesmo com o aumento da produção de lactato sanguíneo ${ }^{5}$.

Segundo alguns estudos, concentrações de lactato podem variar em média de 5 a 7 $\mathrm{mmol} / \mathrm{L}$. Essa produção indica a magnitude da capacidade de produção de ATP via metabolismo 
anaeróbico lático, e remoção para o sangue com a finalidade de regular o $\mathrm{pH}$ intramuscular ${ }^{3,4}$.

A remoção do lactato sanguíneo pode ser direcionada a reutilização deste substrato como fonte energética em órgãos como fígado, coração e tecido muscular, mais especificamente palas fibras de concentração lenta (tipo I) ${ }^{6}$. 0 aumento dos níveis de lactato e prótons de hidrogênio produzidos no músculo podem estar relacionados à queda de rendimento do atleta, levando à fadiga e consequentemente, ao aumento da acidose intramuscular.

O termo "fadiga muscular" é a diminuição da capacidade de manter geração da força e velocidade de relaxamento, indicando alterações nas características contráteis do músculo e alterações nas propriedades elétricas que geram disfunções no sistema neuromuscular humano. Tentando minimizar tais efeitos, várias estratégias foram difundidas no meio desportivo com a finalidade de acelerar o processo de recuperação muscular pós-exercício ${ }^{7,8}$.

Apesar dessas técnicas estarem em uso atualmente, não existe consenso na literatura a respeito do melhor método para a recuperação pós esforço. Tais elementos são provenientes principalmente da grande variação de aplicações, como temperatura da água, tempo de exposição do indivíduo, condição física específica do atleta e parâmetros fisiológicos utilizados.

Dentre as diversas técnicas de recuperação, o contraste passa a ser uma forma aparentemente efetiva na aceleração da recuperação pós-esforço. Segundo Pastre et al. ${ }^{10}$, a técnica de contraste consiste na alternância de exposição ao frio e ao calor, com o intuito de aumentar o metabolismo e auxiliar na recuperação física.

No esporte, tem sido utilizada nos processos de recuperação visando à remoção de lactato sanguíneo e diminuição de marcadores de lesão como a creatina quinase (CK). Seus possíveis efeitos são a pressão hidrostática exercida pela imersão em água e o efeito vasoconstritor e vasodilatador causado pela técnica ${ }^{10}$.

Nesse sentido, a técnica de contraste tem como finalidade acelerar o processo de remoção de lactato e contribuir na recuperação de atletas. No entanto, não existe consenso na literatura sobre seus efeitos. Desse modo, o objetivo deste estudo foi verificar os efeitos da técnica de contraste sobre as concentrações plasmáticas de lactato durante a recuperação pós-esforço em atletas de voleibol.

\section{METODOLOGIA}

Sujeitos

A amostra foi constituída por 18 jogadoras de voleibol, categoria juvenil, com idade entre 17 e 18 anos, residentes na cidade de Presidente Prudente - SP, com tempo de prática mínimo superior a um ano. As voluntárias foram distribuídas de forma randomizada em dois grupos distintos, Grupo controle (GC) e Grupo Tratado (GT).

\section{Desenho experimental}

O GC foi submetido a um protocolo de indução de hiperlactacidemia descrito por Zemková e Hamar ${ }^{11}$, imediatamente após este processo, permaneceram na posição sentada em repouso por período de $25 \mathrm{~min}$, no qual foram coletadas quatro amostras de sangue nos seguintes momentos: Repouso, PÓS-5, PÓS-15 e PÓS-25 min.

O GT passou pelo mesmo protocolo de indução de hiperlactacidemia, porém foram submetidas ao tratamento de contraste por um período de 16 min de imersão alternada em água fria, por $60 \mathrm{seg}$, e água quente, por $120 \mathrm{seg}$. Durante este período foram coletadas quatro amostras de sangue para análise das concentrações de lactato sanguíneo de forma semelhante ao protocolo descrito anteriormente (Repouso, PÓS-5, PÓS-15 e PÓS-25 min).

O presente estudo foi aprovado pelo comitê de ética em pesquisa da Universidade do Oeste Paulista- UNOESTE, № CAAE 28756914.1.0000.5515, todos os envolvidos assinaram um termo de consentimento livre e esclarecido.

Protocolo de indução à hiperlactacidemia

Cada participante foi submetida a aquecimento constituído de cinco minutos de exercícios em cicloergômetro Embreex ${ }^{\circledR} 362$ sem carga. Para induzir o aumento das concentrações sanguíneas de lactato sanguíneo, foi realizada uma versão adaptada de corrida atada em solo durante 30 seg "all-out" da versão original proposta por Zemková e Hamar $^{11}$, que foi realizado em esteira durante $30 \mathrm{seg}$ com intervalo de $15 \mathrm{seg}$. Em seguida, foram realizados saltos laterais sobre um cone plástico com $15 \mathrm{~cm}$ de altura "all-out", por mais $30 \mathrm{seg}$. Não foram 
registrados valores de velocidade máxima, mínima ou índice de fadiga, visto que o objetivo do protocolo criado é apenas induzir aumento das concentrações plasmáticas de lactato sanguíneo.

\section{Protocolo de contraste}

As participantes foram imergidas em água em nível da espinha ilíaca, antero-superior, em posição ortostática, alternando em dois recipientes de plástico com capacidade de $260 \mathrm{~L}$, sendo primeiro por $60 \mathrm{seg}$ em água fria $\left(10^{\circ} \mathrm{C}\right)$ e em seguida por $120 \mathrm{seg}$ em água quente $\left(42^{\circ} \mathrm{C}\right)$ durante $15 \mathrm{~min}$, seguindo o modelo proposto por Coffey et al. ${ }^{12}$.

Para análise do lactato sanguíneo foram coletadas amostras de sangue $(25 \mu \mathrm{L})$, utilizando materiais descartáveis (luvas, algodão e lancetas), do lóbulo direito da orelha, por meio de capilar sanguíneo e injetadas no lactímetro portátil da marca ACCUTREND ${ }^{\circledR}$ PLUS para a determinação da concentração plasmática ( $\mathrm{mmol} / \mathrm{L}$ ).

Análise estatística

Os resultados foram apresentados em média e desvio padrão (DP). Após a obtenção dos dados, foi verificada a normalidade pelo teste Shapiro-Wilk e em seguida utilizado o teste $t$ de Student, para comparação entre médias dos grupos GC e GT, nos diferentes momentos (Repouso, PÓS-5, PÓS-15 e PÓS-25 min). Para tal, foi utilizado o software com pacote estatístico SPSS $22.0^{\circledR}$ for Windows, estabelecendo valores de significância de $p<0,05$.

\section{RESULTADOS}

Os resultados expostos na Tabela 1 representam as características antropométricas das voluntárias que fizeram parte da amostra deste estudo.
Tabela 1. Idade, peso corporal, estatura e índice de massa corporal (IMC) (média \pm DP) do Grupo Controle (GC) e Grupo tratado (GT).

\begin{tabular}{lcc}
\hline \multicolumn{1}{c}{ Variável } & $\begin{array}{c}\mathrm{GC} \\
(\mathrm{N}=9)\end{array}$ & $\begin{array}{c}\mathrm{GT} \\
(\mathrm{N}=9)\end{array}$ \\
\hline Idade & $18,44 \pm 0,72$ & $18,44 \pm 0,72$ \\
Peso $(\mathrm{Kg})$ & $76,1 \pm 23,58$ & $66,35 \pm 14,86$ \\
Estatura $(\mathrm{m})$ & $1,70 \pm 0,12$ & $1,66 \pm 0,07$ \\
$\mathrm{IMC}\left(\mathrm{Kg} / \mathrm{m}^{2}\right)$ & $24,04 \pm 5,09$ & $24,38 \pm 4,76$ \\
\hline
\end{tabular}

Após a realização das analises, observou-se a concentração média de lactato sanguíneo em repouso de $1,97 \pm 0,3 \mathrm{mmol} / \mathrm{L}$ no grupo GC e $1,63 \pm 0,6 \mathrm{mmol} / \mathrm{L}$ no grupo $\mathrm{GT}$. Na coleta PÓS-5, o GC apresentou valor médio de $8,75 \pm 1,5 \mathrm{mmol} / \mathrm{L}$, enquanto o $\mathrm{GT}$ obteve valor médio de $7,35 \pm 4,1 \mathrm{mmol} / \mathrm{L}$.

Já no período PÓS-15, o GC apresentou valor médio de $8,53 \pm 1,6 \mathrm{mmol} / \mathrm{L}$, enquanto o GT obteve valor médio de $5,3 \pm 1,9 \mathrm{mmol} / \mathrm{L}$. Por fim, no período PÓS-25, o GC apresentou valor médio de $6,87 \pm 2,1 \mathrm{mmol} / \mathrm{L}$, enquanto o GT obteve valor médio de $3,33 \pm 1,47 \mathrm{mmol} / \mathrm{L}$.

Foram observadas diferenças estatisticamente significantes $(p<0,05)$ em todos os momentos no grupo GT em comparação ao grupo GC (Figura 1).

Foi possível verificar também, os valores de concentração de lactato sanguíneo nos diferentes momentos da recuperação (Figura 2). 

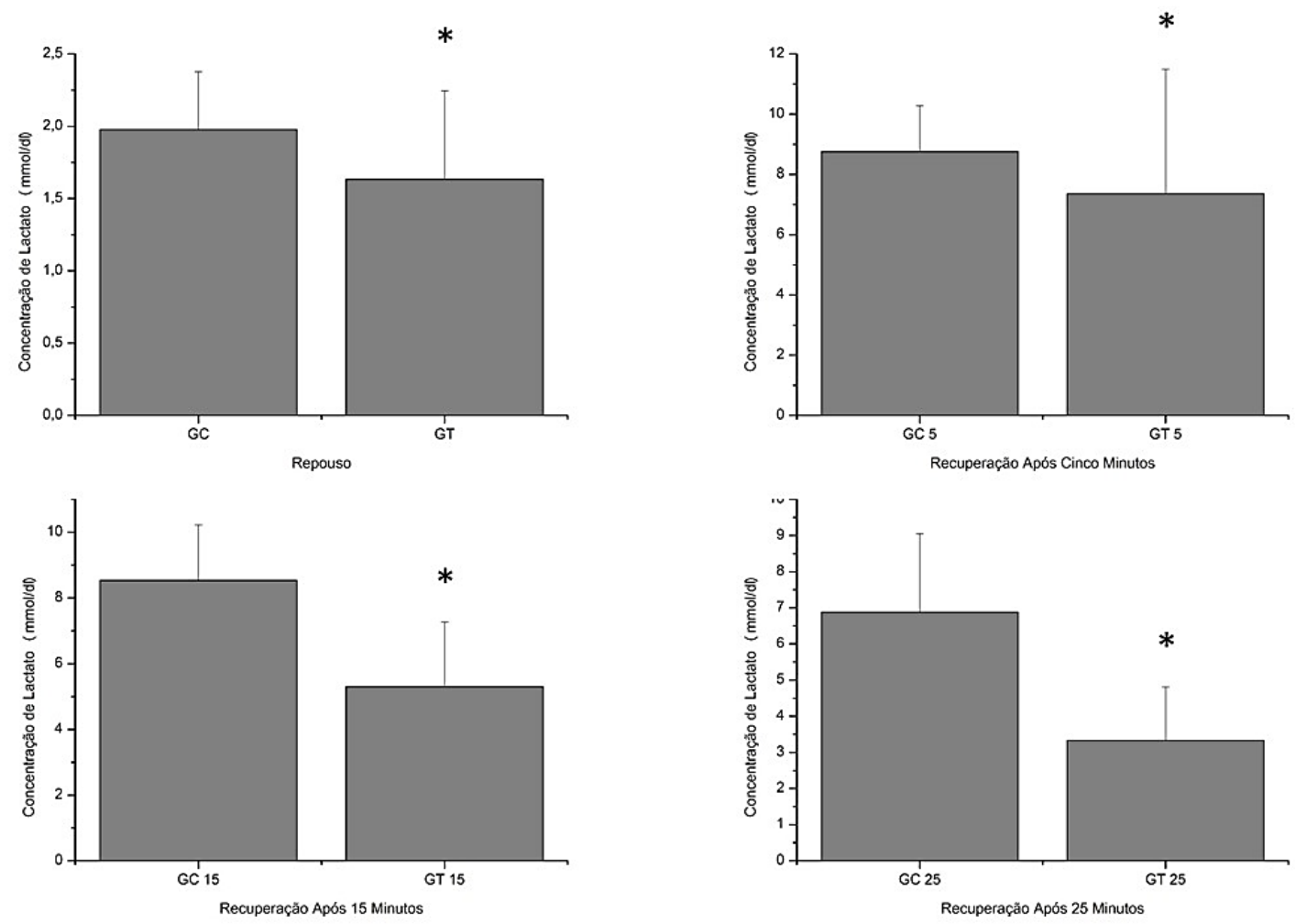

Figura 1. Concentração de lactato sanguíneo em repouso, após 5,15 e 25 min de recuperação. * p<0,05 em comparação ao GC.



Figura 2. Concentração de lactato sanguíneo nos diferentes momentos da recuperação.

DISCUSSÃO

Colloq Vitae2017 set-dez; 9(3): 24-30. DOI: 10.5747/cv.2017.v09.n3.v205

ISSN 1984-6436/C 2017 - Publicado pela Universidade do Oeste Paulista.

Artigo Open Access sob uma licença CC BY-NC-ND (http://creativecommons.org/licenses/by-nc-nd/4.0/).
O objetivo deste estudo foi investigar os efeitos da técnica contraste na recuperação pós- 
esforço sobre a concentração de lactato sanguíneo em jogadoras de voleibol. Os resultados mostram que a técnica resultou na diminuição das concentrações de lactato sanguíneo no grupo GT, nos momentos PÓS-5, PÓS-15 e PÓS-25 comparado ao GC, assim demonstrando sua eficácia na remoção de lactato após esforço.

Segundo Ferrari ${ }^{13}$, a recuperação com crioterapia e a recuperação passiva demonstram menor remoção do lactato sanguíneo quando comparadas à recuperação ativa. No entanto, no presente estudo, foi verificado que a técnica de contraste no GT demonstrou menores valores da concentração de lactato sanguíneo em todos os momentos analisados comparado ao $G C(p<0,05)$.

A implicação prática da técnica de contraste pode ser verificada no meio desportivo, pois a queda no desempenho anaeróbio pode estar relacionada a fatores como microlesões na estrutura contrátil do músculo esquelético, conhecida como dano muscular ${ }^{14}$ e ao acúmulo de $\mathrm{H}+$, favorecendo, portanto, a queda do $\mathrm{pH}$ intracelular ${ }^{15}$. Além disso, outros fatores como a depleção de substratos (principalmente glicogênio muscular), hipertermia e acúmulo de vários metabólitos, entre eles: adenosina difosfato (ADP), fosfato inorgânico (Pi), amônia $(\mathrm{NH} 3)$ e espécies reativas de oxigênio $(\mathrm{ROS})^{15}$ podem estar relacionados à fadiga resultante do exercício de alta intensidade.

No caso do presente estudo, foi utilizado o exercício em bicicleta ergométrica para indução à hiperlactacidemia. Nesse sentido, pesquisas de Serresse ${ }^{16}$ e $\mathrm{Granir}^{17}$, com exercício de alta intensidade realizado durante o Teste de Wingate, ocorrem contribuições de $23 \%$ do metabolismo aeróbio, $49 \%$ do metabolismo glicolítico (anaeróbio lático) e $28 \%$ do sistema ATP-CP (anaeróbio alático) ${ }^{18}$, que leva ao estado de acidose devido aumento do $\mathrm{H}+$ e alteração no pH. No entanto, a contribuição de cada um dos sistemas energéticos parece ser diferente conforme o gênero (masculino ou feminino) e o tipo de treinamento a que é submetido.

Apesar de existir o questionamento sobre a validade da concentração de lactato como parâmetro para determinar a recuperação muscular pós-exercício ${ }^{18}$, tal método tem sido amplamente utilizado para esta finalidade. Para Robergs $^{19}$, o acúmulo de lactato pode ser um indicador indireto do incremento do próton $\mathrm{H}+\mathrm{e}$ a diminuição do pH celular e sanguíneo (fatores promotores da acidose metabólica).

Os mecanismos exatos relacionados aos fenômenos da fadiga, assim como a participação do ácido lático neste processo, não estão completamente elucidados ${ }^{20,21}$, entretanto, sugere-se que, ao passo que uma técnica de recuperação aumenta a remoção de lactato, esta pode influenciar na recuperação. Logo, quanto maior eficácia de remoção destas substâncias pela circulação sanguínea, mais rapidamente o músculo retorna ao seu estado basal, o que teoricamente o torna mais apto para realizar uma nova atividade, com dispêndio menor de tempo ${ }^{22,23}$.

A utilização da crioterapia é outra técnica de recuperação amplamente difundida, especialmente entre atletas de elite. Em estudos que aplicaram tal procedimento, observa-se variação desde 10 até 193 min e temperatura de $1^{\circ} \mathrm{C}$ a $15^{\circ} \mathrm{C}$. Na prática desportiva, a efetividade da técnica para fins de recuperação muscular pós-exercício ainda carece de evidências científicas $^{8,23}$.

$$
\text { Estudos de } \text { Morton }^{9} \text { e Gill }{ }^{22}
$$
demonstram a efetividade da técnica de contraste, ratificando a informação de que o contraste acelera a remoção de catabólitos produzidos durante esforço de alta intensidade. Além disso, descreveram o relaxamento da musculatura esquelética e melhora da percepção subjetiva de recuperação com a aplicação da técnica.

Tratando-se mais especificamente da técnica, alguns autores puderam concluir que a acelerada remoção do ácido lático se deve ao efeito de vasoconstrição e vasodilatação promovido pela técnica ${ }^{23,24}$. Além desses efeitos, supõem-se que a pressão hidrostática da água pode influenciar na remoção desses catabólitos $^{21,24}$. Nesse sentido, é importante ressaltar que, quanto mais rápida a eliminação do lactato sanguíneo, mais eficaz a recuperação ${ }^{25,26}$.

Dessa maneira, o presente estudo colabora com a literatura ao verificar o efeito da terapia de contraste na recuperação pós esforço. Dentre as limitações do presente estudo, destaca-se a análise da recuperação por meio das concentrações de lactato sanguíneo. Por fim, sugere-se que futuros estudos, correlacionem uma maior quantidade de variáveis fisiológicas como lactato sanguíneo, enzimas marcadoras de lesão, sensação subjetiva do atleta, desempenho 
subsequente, dor muscular tardia e creatina quinase (CK).

\section{CONCLUSÃO}

Conclui-se que o contraste por imersão, nos parâmetros adotados, mostrou menores valores sobre as concentrações plasmáticas de lactato durante a recuperação pós-esforço em atletas de voleibol.

\section{CONFLITO DE INTERESSE}

Os autores declaram não haver qualquer potencial conflito de interesse que possa interferir na imparcialidade deste trabalho científico.

\section{REFERÊNCIAS}

1. Brum PC, Forjaz LM, Tinucci T, Negrão CE. Adaptações agudas e crônicas do exercício físico no sistema cardiovascular. Rev Paul Educ Fís. 2004;18(21):21-31.

2. Camargo Filho JCS. Garcia BC, Kodama FY, Bonfim MR, Vanderlei LCM, Ramos EMC, Camargo RCT, Padulla SAT, Maeda JK. Efeitos do exercício aeróbio no músculo esquelético de ratos expostos à fumaça de cigarro. Rev Bras Med Esporte. 2011;17(6):416-9. DOI: https://doi.org/10.1590/S1517-86922011000600010

3. Forjaz CLM, Tricoli V. A Fisiologia em educação física e esporte. Rev Bras Educ Fís. Esporte. 2011;25(7):7-13.

4. Barnett A. Using recovery modalities 1 . between training sessions in elite athletes. Does it help? Sports Med. 2006;36(9):781-96. DOI: https://doi.org/10.2165/00007256-200636090-00005

5. Kustrup P, Zebis M, Jensen JM, Mohr M. Gameinduced fatigue patterns in elite female soccer. J Strength Cond Res 2010;24:437-41. DOI: https://doi.org/10.1519/JSC.0b013e3181c09b79

6. Krustrup P, Mohr M, Steensberg A, Bencke J, Kjaer $\mathrm{M}$, Bangsbo J. Muscle and blood metabolites during a soccer game: implications for sprint performance. Med Sci Sports Exerc 2006;38:1165-74. DOI: https://doi.org/10.1249/01.mss.0000222845.89262.cd

7. Foschini D, Prestes J, Mário AC. Relação entre exercício físico, dano muscular e dor muscular de inicio tardio. Rev Bras Cineantropom Desempenho Hum. 2007;9(1):101-6.

8. Baroni BM, Leal junior ECP, Senerosi RA, Grosselli G, Censi S, Bertolla F. Efeito da Crioterapia de Imersão

Colloq Vitae2017 set-dez; 9(3): 24-30. DOI: 10.5747/cv.2017.v09.n3.v205 ISSN 1984-6436/@ 2017 - Publicado pela Universidade do Oeste Paulista. Artigo Open Access sob uma licença CC BY-NC-ND (http://creativecommons.org/licenses/by-nc-nd/4.0/). sobre a Remoção do Lactato Sanguíneo após Exercício. Rev Bras Cineantropom Desempenho Hum. 2010;12(3):179-85.

9. Morton RH. Contrast water immersion hastens plasma lactate decrease after intense anaerobic exercise. J Sci Med Sport. 2007;10(6):467-70. DOI: https://doi.org/10.1016/i.jsams.2006.09.004

10. Pastre CM, Bastos FN, Junior JN, Vanderlei LCM, Hoshi RA. Métodos de recuperação pós-exercicio: uma revisão sistemática. Rev Bras Med Esporte. 2009;15(2):138-44. DOI: https://doi.org/10.1590/S1517-86922009000200012

11. Zemková E, Hamar D. "All-out" tethered running as an alternative to Wingate anaerobic test. Kinesiol Rev. 2004;36(2):165-72.

12. Coffey V, leveritti M, Gill N. Effect of recovery modality on 4-hour repeated treadmill running performance and changes in physiological variables. J Sci Med Sport. 2004;7(1):1-10. DOI: https://doi.org/10.1016/S1440-2440(04)80038-0

13. Ferrari HG, Oliveira R, Strapasson MV, Santa Cruz RAR, Libardi CA, Cavaglieri CR. Effect of different methods of recovery on the lactate removal and anaerobic performance in soccer players. Rev Bras Med Esporte. 2013;19(6):423-6. DOI: https://doi.org/10.1590/S1517-86922013000600009

14. Clarkson PM, Hubal MJ. Exercise-induced muscle damage in humans. Am J Phys Med Rehabil 2002;81:S52-69. DOI: https://doi.org/10.1097/00002060-200211001-00007

15. Lamb GD. Point: counterpoint: lactic acid accumulation is an advantage/disadvantage during muscle activity. J Appl Physiol 2006;100:1410-2. DOI: https://doi.org/10.1152/japplphysiol.00023.2006

16. Serresse O, Lortie G, Bouchard C, Boulay MR. Estimation of the contribution of the various energy systems during maximal work of short duration. Int J Sports Med. 1988;9(6):456-60. DOI: https://doi.org/10.1055/s-2007-1025051

17. Granier P, Mercier B, Mercier J, Anselme F, Préfaut C. Aerobic and anaerobic contribution of Wingate test performance in sprint and middle-distance runners. Eur J Appl Physiol. 1995;70(1):58-65. DOI: https://doi.org/10.1007/BF00601809

18. Hill DW, Smith JC. Gender difference in anaerobic capacity: role of aerobic contribution. Br J Sports Med 1993;27(1):45-48. DOI: https://doi.org/10.1136/bjsm.27.1.45

19. Robergs RA, Ghiasvand F, Parker D. Biochemistry of exercise-induced metabolic acidosis. Am J Physiol 
Regul Integr Comp Physiol. 2004;287(3):502-16. DOI: https://doi.org/10.1152/ajpregu.00114.2004

20. Koutedakis $Y$, Sharp NC. Lactic acid removal and heart rate frequencies during recovery after strenuous rowing exercise. Br J Sports Med 1985;19(4):199-202. DOI: https://doi.org/10.1136/bjsm.19.4.199

21. Cairns SP. Lactic acid and exercise performance: culprit or friend? Sports Med 2006;36(4):279-91. DOI: https://doi.org/10.2165/00007256-200636040-00001

22. Gill ND, Beaven CM, Cook C. Effectiveness of postmatch recovery strategies in rugby players. Br J Sports Med. 2006;40(3):260-3. DOI: https://doi.org/10.1136/bjsm.2005.022483

23. Wilcock IM, Cronim JB, Hing WA. Physiological response to water immersion: a method for sport recovery? Sports Med. 2006;36(9):747-65. DOI: https://doi.org/10.2165/00007256-200636090-00003

24. Baroni BM, Leal Junior ECP, Generosi RA, Grosselli G, Censi S, Bertolla F. Efeito da crioterapia de imersão sobre a remoção do lactato sanguíneo após exercício. Rev Bras Cineantropom Desempenho Hum. 2010, 12(3):179-185.

25. Cochrane DJ. Alternating hot and cold water immersion for athlete recovery: a review. Phys Ther Sports. 2004;5:26-32. DOI: https://doi.org/10.1016/i.ptsp.2003.10.002

26. Connolly DAJ, Brennan KM, Lauzon CD. Effects of active versus passive recovery on power output during repeated bouts of short term, high intensity exercise. J Sports Sci Med. 2003;2:47-51.

Recebido para publicação em 15/08/2017

Revisado em 11/09/2017

Aceito em 13/09/2017 